Revistade
Economila
Contemporâned

\title{
ANÁLISE DA RELAÇÃO DE CURTO E LONGO PRAZOS ENTRE AS POLÍTICAS MONETÁRIA E FISCAL COM CRESCIMENTO ECONÔMICO NO BRASIL: APLICAÇÃO DE MODELOS VEC
}

\author{
Emerson Costa dos Santos ${ }^{a}$ \\ Maria Alice Ferreira ${ }^{b}$ \\ João Eustáquio de $\operatorname{Lima}^{c}$ \\ André Luís Mota dos Santos ${ }^{d}$
${ }^{a}$ Estudante do Doutorado em Economia Aplicada da Universidade Federal de Viçosa (UFV).

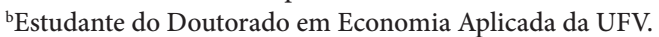
'Professor do Departamento de Economia Rural da UFV.
${ }^{\mathrm{d}}$ Departamento de Economia da Universidade Federal da Bahia. \\ Artigo recebido em 14/07/2014 e aceito em 17/09/2015.

\begin{abstract}
RESUMO: O objetivo do artigo foi verificar empiricamente a relação de curto e longo prazo entre as políticas monetárias e fiscais com o crescimento econômico no Brasil no período recente após a implantação do sistema de metas de inflação. A questão se justifica na medida em que os desequilíbrios macroeconômicos vividos no Brasil recentemente podem ser explicados por ausência de coordenação entre as políticas monetária e fiscal. Investiga-se a relação de equilíbrio de longo prazo com a metodologia de modelos de correção de erros vetorial (VEC). Os resultados ilustraram a importância das variáveis de política monetária e fiscal na explicação de variações do PIB brasileiro na relação de longo prazo.
\end{abstract}

PALAVRAS-CHAVE: longo prazo; curto prazo; modelo VEC.

CLASSIFICAÇÃO JEL: E6; C220.

Correspondência para: Emerson da Costa dos Santos

Contato: emersoco@hotmail.com 


\title{
ANALYSIS OF THE RELATIONSHIP BETWEEN SHORT AND LONG TERMS MONETARY AND FISCAL POLICY WITH ECONOMIC GROWTH IN BRAZIL: APPLICATION OF MODELS VEC
}

\begin{abstract}
This paper aims to assess the relationship between short and long term monetary and fiscal policies, on the one hand, and economic growth in Brazil in recent years, on the other, after the implementation of inflation targeting system. This research question is justified to the extent that the recent macroeconomic imbalances lived in Brazil can be explained by the lack of coordination between monetary and fiscal policies. This study investigates the relationship between long-term equilibrium and the methodology of different vector error correction (VEC). The results illustrate the importance of monetary and fiscal policies as explanatory variables for the variations in the Brazilian GDP in the long term.
\end{abstract}

KEYWORDS: long run; short run; VEC model. 


\section{INTRODUÇÃO}

A inconsistência entre as políticas fiscal e monetária pode ser uma das possíveis explicações para os desequilíbrios macroeconômicos sistemáticos internos e/ou externos que a economia brasileira tem apresentado nos últimos anos. No período mais recente, após a implantação do plano Real, tanto a política monetária quanto a evolução da dívida pública brasileira têm sido muito discutidas no meio acadêmico e pelas autoridades de política. A primeira destacou-se ao obter sucesso no combate à inflação apesar de contratempos, e a segunda apresentou elevação no período em termos nominais, mas declínio quando medida como proporção do Produto Interno Bruto (PIB).

Os estudos acerca da combinação entre política fiscal e política monetária sempre estiveram no centro do debate macroeconômico. Sargent e Wallace (1981) argumentam que a política monetária pode ser completamente ineficaz no combate à inflação caso a política fiscal não seja sustentável.

Até recentemente, estudos sobre a análise da dinâmica macroeconômica enfatizavam apenas os choques de política monetária, negligenciando o papel da política fiscal, fato que, segundo Perotti (2002), constitui um infortúnio, uma vez que há muito mais dispersão de crenças entre os economistas sobre os efeitos da política fiscal sobre a economia se comparado com aqueles relacionados à política monetária.

No entanto, recentemente, tanto na prática quanto na teoria têm-se evidenciado crescente ligação entre política monetária e fiscal e crescimento econômico. Diversos autores, entre os quais Sims (2003) e Favero (2004), argumentam que um efetivo sistema de metas de inflação depende da austeridade fiscal, ou seja, de uma política fiscal cujo superavit primário seja frequentemente ajustado de tal forma que a proporção da dívida em relação ao produto seja mantida constante.

Ao lado da política fiscal, Pastore (1995) argumenta que em um contexto de restrição de liquidez, a parcela da dívida pública atrelada à taxa Selic (conhecida como Letras Financeiras do Tesouro - LFT) pode gerar efeito renda e efeito riqueza em um contexto em que a importância da gestão da dívida pública é crucial na discussão da eficácia da política monetária no Brasil. Quanto ao primeiro efeito, quando a taxa de juros se eleva, os fluxos de rendimentos dos detentores dos títulos aumentam, elevando a renda disponível, e gera um efeito positivo no consumo, estimulando a demanda agregada. Quanto ao segundo efeito, este pode não ser gerado, em função de grande parte da dívida ser indexada à taxa de juros de curto prazo. Segundo Amaral e Oreiro (2008), esse efeito está comprometido pela excessiva participação na dívida pública de títulos pós-fixados e sem risco de taxa de juros. Assim, as variações necessárias na taxa de juros seriam maiores do que as que ocorreriam se o efeito riqueza operasse em maior magnitude. Como não existe risco, as variações da taxa de juros 
atuariam no sentido de criar um efeito renda positivo, o que diminuiria ainda mais o poder dos juros como instrumento de política monetária. Portanto, uma fração da riqueza privada se mantém imune à política monetária, reduzindo seu efeito sobre o consumo.

O alcance de metas macroeconômicas, a estabilidade do nível de preços e o crescimento econômico elevado e sustentável além do equilíbrio externo têm sido algumas das prioridades de política econômica, seja de economia desenvolvida ou em desenvolvimento, dada à suscetibilidade de flutuações das variáveis macroeconômicas. Dessa forma, as políticas fiscais e monetárias são as ferramentas importantes por meio das quais uma economia é regulada pelo governo e pelo banco central. Geralmente, tanto as políticas fiscais quanto as monetárias procuram alcançar relativa estabilidade macroeconômica. O impacto relativo de política fiscal e monetária tem sido cada vez mais estudado ao longo do tempo (Musa e Asare, 2013).

Este artigo está organizado em mais quatro seções além desta introdução. Na próxima, contextualizam-se as relações entre as políticas monetária e fiscal. Na terceira, apresenta-se a metodologia. Na quarta, faz-se a discussão dos resultados. Por fim, na última seção, as considerações finais são apresentadas.

\section{RELAÇÕES ENTRE POLÍTICAS MONETÁRIA E FISCAL}

A teoria macroeconômica de orientação monetarista apresentou uma autoridade monetária que buscava prioritariamente controlar o nível de preços, assumindo que a dívida do governo sempre seria solvente. A utilização da política monetária de forma discricionária seria capaz de suavizar os ciclos econômicos. Na percepção de Friedman, os agentes possuem expectativas adaptativas, e nesse sentido, um choque de política monetária teria algum efeito no curto prazo. Entretanto, para Lucas, um choque de política monetária não teria nenhum efeito, mesmo no curto prazo, devido aos agentes anteciparem o choque de política monetária por meio das expectativas racionais. Nessa linha teórica destacam-se os trabalhos de Kydland e Prescoott (1977), Lucas (1983), Sargent e Wallace (1981) e Sargent (1982). Por outro lado, a literatura de orientação mais intervencionista, ou keynesiana, destacou a importância das políticas discricionárias. Contudo, para superar a crítica dos monetaristas às políticas discricionárias, os modelos novos keynesianos foram refinados por meio da introdução de fundamentos microeconômicos, tais como expectativas racionais e rigidez de preços.

Nessa linha de pensamento, os trabalhos de Leeper (1991), Taylor (1993), Sims (1994) e Woodford (1995), entre outros, foram os precursores. Entretanto, a política fiscal era postergada ao segundo plano; assumia-se um regime ricardiano, em que o 
orçamento do governo estava sempre em equilíbrio, sujeito apenas a variações cíclicas, e qualquer desajuste seria corrigido via tributos ou imposto inflacionário. Tendo como hipótese subjacente a equivalência ricardiana, na qual a autoridade fiscal é bem disciplinada, ou seja, o corte dos impostos do governo financeiro por aumentos no endividamento deverá ser compensado com o aumento de impostos no futuro para garantir que a dívida seja solvente. Nesse contexto, a discussão da coordenação entre as políticas fiscal e monetária não fazia sentido.

Sargent e Wallace (1981), em um artigo seminal acerca da coordenação entre as políticas fiscal e monetária, chamaram atenção para a possibilidade de a autoridade monetária tornar-se incapaz de controlar a inflação. Nesse trabalho, os autores sugerem que a economia pode estar funcionando sobre duas formas: dominância monetária ou dominância fiscal. O primeiro caso trataria de uma situação em que a autoridade monetária determina a quantidade de receita que será ofertada pela autoridade fiscal por meio de senhoriagem para o período presente e todos os subsequentes. A autoridade fiscal, assim, tem os gastos restringidos pela função de demanda por títulos, sendo necessário um superavit que mantenha constante a relação dívida líquida do setor público sobre o PIB (DLSP/PIB). Dentro dessa situação de coordenação entre as políticas econômicas, a autoridade monetária teria o controle sobre a inflação, que seria determinada pela oferta de moeda. Na segunda situação, a autoridade fiscal poderia atuar de forma independente do seu orçamento, de tal forma que anunciasse todos os possíveis deficits e superavits em cada ponto do tempo. Nesse caso, a autoridade fiscal não vincularia seu superavit (ou deficit) à manutenção da relação DLSP/ PIB, em que a expansão de tal razão pode ser financiada pela emissão de títulos ou pela autoridade monetária por meio da senhoriagem.

Tais maneiras de financiar os deficits públicos geram efeitos inflacionários, primeiramente de maneira direta e, posteriormente, de maneira indireta, o que configurar-se-á, nas palavras de Sargent e Wallace (1981), na "aritmética monetarista desagradável”. Essa análise sugere que a submissão da política monetária à política fiscal resulta em inflação, explicitando um importante limite da política monetária em controlar o nível de preços.

Recentemente, surgiram vários trabalhos na literatura com uma visão alternativa, em que apenas um banco central independente não seria suficiente para garantir a estabilidade de preços. Além de uma política monetária apropriada, seria necessária também uma política fiscal apropriada capaz de evitar a inflação. Trata-se da Teoria Fiscal do Nível de Preços (TFNP). Seguindo as interpretações estabelecidas em Cochrane (1998, 2001), Sims (1994) e Woodford (1994, 1995, 2001), tem-se que a ferramenta padrão da política monetária na abordagem monetarista (controle da oferta de moeda) não é suficiente para o controle do nível de preços. Sobre essa ótica, a determi- 
nação do nível de preços é atribuída à política fiscal por meio da taxa de crescimento dos títulos públicos.

A diferença entre a abordagem da TFNP e a de Sargent e Wallace (1981) está na forma com que a restrição orçamentária intertemporal do setor público é interpretada, de acordo com a qual o valor do estoque da dívida deve ser igual ao valor presente, descontados os superavits futuros. O que é visto pela abordagem tradicional como uma restrição orçamentária intertemporal é visto pela TFNP como uma condição de equilíbrio, em que um determinado nível de preços iguala o valor real das obrigações do governo ao valor presente dos superavits. A TFNP destacou que, se a solvência do governo não é garantida, a política monetária não será capaz de controlar o nível de preços. Por outro lado, a fim de garantir a estabilidade, a política fiscal tem de reagir suficientemente forte para conter um aumento na taxa de juros em caso de pressões inflacionárias pelo aumento do superavit primário. Em outras palavras, a política monetária, com o objetivo de manter a inflação em check, precisa estar de mãos dadas com a política fiscal, obedecendo a uma política de restrição de solvência.

A TFNP baseia-se em uma política fiscal sobre a hipótese não-ricardiana: os gastos públicos são estabelecidos sem que seja levada em consideração a restrição intertemporal. Na verdade, para a TFNP não se tem mais uma restrição, mas uma condição de equilíbrio (Rocha e Paschoalotto, 2004). Ressalta-se, porém, que na discussão acerca da coordenação entre as políticas fiscal e monetária um aspecto importante perpassa tanto a definição do regime monetário adotado quanto a forma pela qual o deficit público é financiado.

O regime de metas para a inflação, por exemplo, deve tornar as demais variáveis macroeconômicas subordinadas ao uso da taxa nominal de juros, já que esta é a variável de controle à disposição da autoridade monetária para obter um nível de preços convergente com o intervalo socialmente desejado. Isso significa que o tipo de política fiscal para uma economia que segue o regime de metas inflacionárias tem consequências importantes para o sucesso da política monetária (Junior e Jesus, 2010).

Nesse modelo, devidamente considerando uma economia em que o regime monetário é do tipo metas para a inflação, um aumento da taxa de juros proveniente de pressões inflacionárias aumenta o custo da dívida pública e, em decorrência, a probabilidade de default. Dado o ambiente de aversão ao risco, isso pode acarretar uma fuga de capitais e uma conseguinte desvalorização cambial ao invés de uma valorização. A desvalorização cambial, por sua vez, pode comprometer tanto a meta de inflação quanto a estabilidade das expectativas inflacionárias, o que não é desejável. Se parte expressiva da dívida doméstica estiver denominada em moeda estrangeira, a desvalorização cambial provoca um aumento ainda mais significativo na dívida pública, afetando as expectativas de inflação e completando o ciclo vicioso. De acordo com a 
abordagem de Blanchard (2004), se a política monetária for dominada pela política fiscal, considerando um quadro fiscal perverso, e a dívida fortemente indexada aos juros e à moeda externa, o aumento na taxa de juros pela autoridade monetária não necessariamente reduz a inflação por meio dos canais tradicionais: desestímulo à demanda agregada e apreciação cambial.

A literatura teórica acerca da coordenação das políticas monetária e fiscal tem crescido consideravelmente nos últimos anos. Trabalhos desenvolvidos por Benigno e Woodford (2004), Persson, Persson e Svensson (2004) e Schmitt-Grohe e Uribe (2004a, 2004b, 2006) são alguns exemplos de modelos com características novo-keynesianas que procuram explicar o papel da coordenação ótima das autoridades fiscais e monetárias. Se tal coordenação é observada, significa que ambas as políticas econômicas estarão sob a mesma função objetivo e, por conseguinte, estarão enfrentando a mesma restrição orçamentária do setor público.

Em geral verifica-se que a coordenação entre a política monetária e a fiscal reduz a possibilidade de conflito de interesses entre os responsáveis pela política econômica e a incerteza para a tomada de decisões dos agentes econômicos. Conforme Mendonça (2001), o ganho potencial a partir da coordenação é elevado. E, portanto, uma política de coordenação macroeconômica deveria ser estruturada de forma que a reação da taxa de juros seja capaz de compensar a contração fiscal.

Nos últimos anos, a eficácia relativa de ação da política monetária e fiscal no crescimento econômico vem sendo debatida por ambos os economistas formuladores de políticas macroeconômicas. A literatura tradicional nas áreas de políticas monetárias e fiscais ótimas aponta que a interação entre essas políticas tem grande importância para o crescimento econômico.

Nas últimas décadas, o desenvolvimento da literatura de crescimento endógeno iniciada por Romer (1986), Lucas (1988) e Rebelo (1991) tem melhorado a compreensão sobre como o desempenho de crescimento de uma economia pode ser afetado pelas políticas públicas. Barro (1990) e King e Rebelo (1990) estudaram os efeitos das políticas fiscais, tais como os gastos do governo e da tributação, sobre o crescimento econômico. Em resumo, o resultado geral é que a tributação afeta negativamente o desempenho do crescimento no longo prazo e que os impactos quantitativos são muito maiores do que os encontrados em modelos de crescimento exógenos. Chari, Jones e Manuelli (1995) e Ploeg e Alogoskoufis (1994) examinaram os efeitos das políticas monetárias, tais como mudanças nas taxas de crescimento da oferta de moeda nominal, na atividade real de longo prazo. Esses autores encontraram suporte na literatura convencional para indicar que a inflação e o crescimento em longo prazo possuem uma relação negativa. Esses estudos também representam um avanço na compreensão do impacto da alternativa de políticas sobre a inflação e o crescimento. 
A inflação pode reduzir as taxas de investimento da economia, considerando que a variação dos preços funcionaria como uma taxação ao investimento. Além disso, altas taxas de inflação provocam custos sociais que são prejudiciais ao crescimento econômico, afetando a produção e o mercado de trabalho, por meio da decisão entre trabalho e lazer.

\section{METODOLOGIA}

A análise empírica deste trabalho está baseada na Econometria de Séries Temporais e, em especial, na metodologia dos Vetores Autorregressivos (VAR). Com esse método busca-se estimar um ou mais vetores de cointegração entre as variáveis que signifiquem uma relação estável entre elas, interpretadas como uma relação de longo prazo.

De forma geral, um $\operatorname{VAR}(p)$ com $k$ variáveis pode ser representado na forma matricial por:

$$
Y_{t}=\alpha+\theta_{1} Y_{t-1}+\theta_{2} Y_{t-2}+\cdots+\theta_{p} Y_{t-p}+\varepsilon_{t}
$$

Em (1), $y_{t}$ é um vetor $K \times 1$ de variáveis endógenas, $Y_{t-j}, j=1,2,3 \ldots ; p$ são vetores $k x 1$ de variáveis defasadas; $\alpha$ é um vetor $k \times 1$ de interceptos; $\theta_{i}, i=123 \ldots, p$ são matrizes kxk de coeficientes a serem estimados; e $\varepsilon_{1}$ é um vetor kx1 de erros aleatórios com média zero, $E\left(\varepsilon_{t}\right)=0$, e matriz de variâncias e covariâncias $E\left(\varepsilon_{t} \varepsilon^{\prime} t\right)=\Sigma$.

Pela descrição de Bueno (2008), a ideia intuitiva de cointegração é a de que as variáveis não estacionárias podem caminhar juntas, isto é, podem ter trajetórias temporais interligadas, de forma que no longo prazo apresentem relação de equilíbrio

$$
\begin{gathered}
Y_{t}=Y_{t-1}+\varepsilon_{1 t} \varepsilon_{1 t} \sim \operatorname{iid}\left(0, \sigma^{2}\right) \\
X_{t}=X_{t-1}+\varepsilon_{2 t} \varepsilon_{2 t} \sim i i d\left(0, \sigma^{2}\right)
\end{gathered}
$$

À semelhança de Bueno (2008), outros autores afirmam que os casos mais estudados e de maior interesse são aqueles em que as variáveis são integradas de ordem um ou há algumas integradas de ordem 1 (um) e outras de ordem zero. Intuitivamente, a análise de cointegração consiste em verificar se as variáveis guardam uma relação de equilíbrio de longo prazo, isto é, se elas possuem uma dinâmica comum que faz com que caminhem juntas ao longo do tempo.

Dois métodos de cointegração podem ser ajustados a conjuntos de séries de tempo. O primeiro é o procedimento de Engle e Granger (1987), que estima um único vetor de cointegração e permite apenas avaliar a cointegração entre pares de variáveis inte- 
gradas de ordem 1 (um). O segundo procedimento é a cointegração de Johansen (1996), e possui vantagens comparativas em relação ao primeiro procedimento.

O procedimento de Engle e Granger (1987) baseia-se na ideia de que, se duas variáveis I(1) são cointegradas de primeira ordem, então a equação de cointegração pode ser obtida por estimação de mínimos quadrados ordinários. Os resíduos dessa especificação devem ser estacionários.

Partindo da representação de Granger, Johansen (1996) argumenta que, se um grupo de variáveis cointegram entre si, então o vetor de cointegração tem uma representação na forma de um modelo de correção de erro. O modelo de correção de erro pode ser considerado uma variação da metodologia Vetor Autorregressivo (VAR) e tem como principal vantagem a não predisposição a caracterizar as variáveis pelo seu grau de endogeneidade, além de descartar o problema de simultaneidade, pois cada variável é explicada pelas próprias defasagens e pelas defasagens das demais:

$$
\begin{gathered}
y_{t}=\beta_{0}+\beta_{1} x_{t}+\varepsilon_{t} \\
\Delta \hat{\varepsilon}_{t}=\gamma \hat{\varepsilon}_{t-1}+\sum_{i=1}^{p} \delta_{i} \Delta^{i} \hat{\varepsilon}_{t-1}+\beta_{1} x_{t}+\xi_{t}
\end{gathered}
$$

As variáveis envolvidas na análise são: $\mathrm{PIB}$, dívida pública, superavit primário, taxa de câmbio, IPCA, expectativa do IPCA, M1. A análise foi realizada para o período pósmetas de inflação. Dessa forma, os dados consistem em observações mensais para o período de julho de 2001 a novembro de 2013, obtidos no Instituto de Pesquisa Econômica e Aplicada (IPEADATA) e no Banco Central do Brasil. Todas as séries foram logaritmizadas. O modelo de longo prazo a ser estimado pode ser escrito da seguinte forma:

$$
\begin{aligned}
& L P I B_{t}=\beta_{0}+\beta_{1} \text { Ldivpib }_{t}+\beta_{2} L S P_{t}+\beta_{3} \text { Lcâmbio }_{t}+\beta_{4} \text { Lipca }_{t}+\beta_{5} \text { Lexpipca }_{t}+ \\
& \beta_{6} L m 1_{t}+\beta_{7} L \text { Selic }_{t}+\varepsilon_{t}
\end{aligned}
$$

O PIB mensal é o PIB acumulado nos últimos 12 meses. A relação dívida/PIB, divpib, constitui a dívida líquida do setor público, compreendendo o Governo Federal e Banco Central do Brasil, governos estaduais, governos municipais e empresas estatais, como porcentagem do PIB. A relação superavit primário/PIB, SP, são as necessidades de financiamento do setor público, formado pelo governo central (governo federal mais o banco central), em porcentagem do PIB. A taxa de câmbio é a taxa de câmbio nominal. O IPCA é a taxa de variação do Índice Nacional de Preços ao Consumidor Amplo, e a expectativa de inflação, expipca, é corresponde à expectativa média de inflação acumulada para os próximos 12 meses. O M1 é a porcentagem dos saldos em fim de período em relação ao PIB. Refere-se à parcela dos meios de pagamentos, que engloba o papel-moeda em poder do público e os depósitos à vista. 


\section{RESULTADOS E DISCUSSÕES}

A fim de avaliar se as variáveis usadas seguem um processo estocástico estacionário, foram realizados quatro testes de raiz unitários. A Tabela 1 mostra os resultados do teste ADF, Dickey-Fuller GLS, PP e KPSS das variáveis em nível.

Tabela 1 - ADF, DF- GLS, PP, KPSS

\begin{tabular}{|c|c|c|c|c|c|c|c|c|}
\hline \multirow[b]{2}{*}{ Variáveis } & \multicolumn{4}{|c|}{ Constante } & \multicolumn{4}{|c|}{ Constante e tendência } \\
\hline & $\begin{array}{c}\text { ADF } \\
\text { t-estat. }\end{array}$ & $\begin{array}{c}\text { DF-GLS } \\
\text { t-estat. }\end{array}$ & $\begin{array}{c}\text { PP } \\
\text { t-estat. }\end{array}$ & $\begin{array}{r}\text { KPSS } \\
\text { t-estat. }\end{array}$ & $\begin{array}{c}\text { ADF } \\
\text { t-estat. }\end{array}$ & $\begin{array}{l}\text { DF-GLS } \\
\text { t-estat. }\end{array}$ & $\begin{array}{c}\text { PP } \\
\text { t-estat. }\end{array}$ & $\begin{array}{r}\text { KPSS } \\
\text { t-estat. }\end{array}$ \\
\hline LPIB & $-1,99$ & 1,10 & $7,74^{*}$ & $1,45^{*}$ & $-1,40$ & $-0,86$ & $-5,44^{*}$ & $0,26^{*}$ \\
\hline LDÍVPIB & $-1,61$ & 0,73 & $-1,58$ & $1,37^{\star}$ & $-4,29^{*}$ & $-1,48$ & $-3,95^{\star *}$ & 0,07 \\
\hline LSP & $-0,20$ & $-1,05$ & $-1,01$ & 0,11 & $-2,45$ & $-1,45$ & $-3,05$ & 0,10 \\
\hline LCAM & $-0,47$ & $-1,57$ & $-0,46$ & $0,97^{\star}$ & $-2,08$ & $-1,99$ & $-2,17$ & $0,20^{* *}$ \\
\hline LIPCA & $-2,12$ & $-4,41^{*}$ & $-2,07$ & $0,53^{\star *}$ & $-3,12$ & $-3,14^{\star *}$ & $-2,88$ & $0,20^{* *}$ \\
\hline LEXPIPCA & $-0,81$ & $-2,68^{*}$ & $-0,78$ & $0,37^{\star * *}$ & $-3,12$ & $-3,14^{* *}$ & $-2,88$ & $0,20^{* *}$ \\
\hline LM1 & 0,36 & $-0,44$ & 1,02 & $1,23^{\star}$ & $-2,54$ & $-2,58$ & $-5,08^{\star}$ & $0,28^{*}$ \\
\hline LSELIC & $-1,33$ & $-0,24$ & $-1,19$ & $1,22^{*}$ & $-3,33^{\star * *}$ & $-2,85^{\star \star *}$ & $-4,20^{*}$ & 0,08 \\
\hline \multicolumn{5}{|c|}{ Valores críticos } & \multicolumn{4}{|c|}{ Valores críticos } \\
\hline $10 \%$ & $-2,57$ & $-1,61$ & $-2,57$ & 0,34 & $-3,14$ & $-2,70$ & $-3,14$ & 0,12 \\
\hline $5 \%$ & $-2,88$ & $-1,94$ & $-2,88$ & 0,46 & $-3,44$ & $-2,99$ & $-3,44$ & 0,14 \\
\hline $1 \%$ & $-3,47$ & $-2,58$ & $-3,47$ & 0,73 & $-4,02$ & $-3,53$ & $-4,02$ & 0,21 \\
\hline
\end{tabular}

Nota: (*) $1 \%$ de significância. (**) 5\% de significância. (***) 10\% de significância.

Fonte: Resultados da pesquisa.

Os resultados dos testes de raiz unitária, apresentados na Tabela 1, indicam que as séries são integradas de primeira ordem, embora não haja unanimidade quanto à rejeição da hipótese nula de raiz unitária para todas as variáveis em nível. Pelo teste KPSS, com hipótese nula de estacionariedade, apenas a variável superavit primário seria estacionária em nível, quando analisada somente com constante. Em primeira diferença, por outro lado, as séries se mostraram estacionárias. A Figura 1 mostra que todas as séries apresentam raiz dentro do círculo unitário invertido.

Assumiu-se, com o amparo de razoável concordância entre a maioria dos testes de raiz unitária, que todas as variáveis do estudo são integradas de primeira ordem, ou seja, possuem uma raiz unitária. Essa descoberta permite a continuidade do procedimento de análise multivariada pelos métodos de cointegração.

Inicialmente foi realizado o modelo VAR irrestrito, para garantir que ele seja uma representação bem especificada dos dados antes da imposição de restrições de cointegração ao modelo. Um requisito importante na condução de testes de cointegração e estimação de um sistema VAR, irrestrito ou restrito no modelo de correção de erros (VEC), é a escolha ideal dos lags. Foram utilizadas duas defasagens no VAR estimado, selecionadas pelos critérios de informação. Por tanto para o VEC será considerado apenas uma lag. 
Figura 1 - Círculo unitário invertido

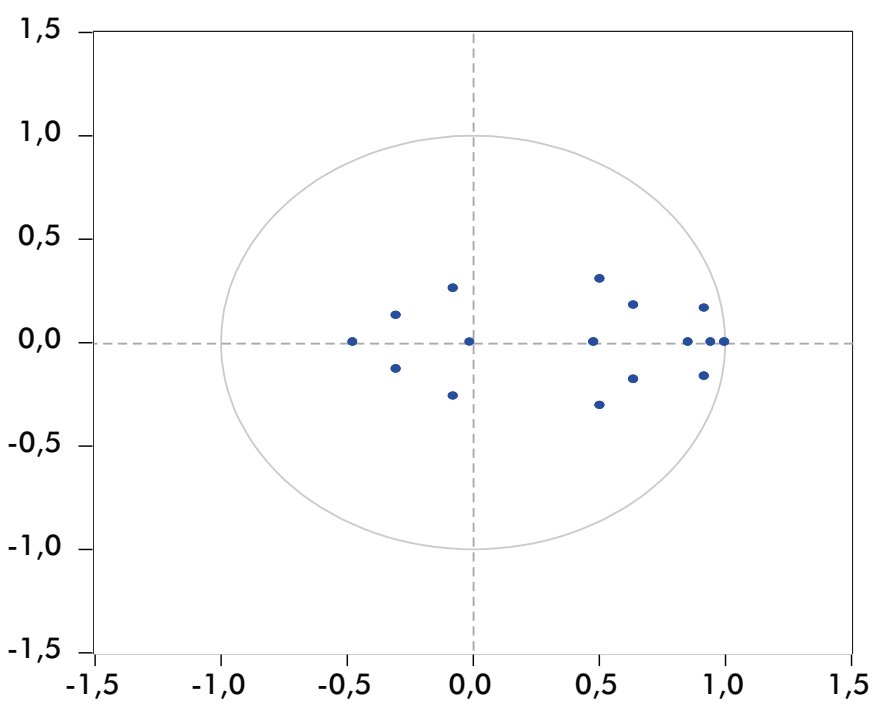

Fonte: Resultados da pesquisa.

Quanto à análise da quantidade de vetores de cointegração, sendo a hipótese nula de que não há nenhum vetor de cointegração versus a hipótese alternativa de que há pelo menos um vetor de cointegração, verificou-se que a hipótese nula foi rejeitada, uma vez que o valor calculado foi superior ao seu respectivo valor de tabela ao nível de $5 \%$. Os valores calculados das estatísticas $\lambda_{\text {traço }}$ indicaram a existência de dois vetores de cointegração e o teste do autovalor máximo $\lambda_{\max }$ indicou a existência de um vetor de cointegração estatisticamente significativo. Portanto, as variáveis apresentam equilíbrio, ou seja, são cointegradas.

Para a presente análise será levada em conta apenas uma relação de longo prazo. Na Tabela 2 são apresentados os coeficientes do vetor de cointegração já normalizado para o PIB e na Tabela 3, os coeficientes do vetor de ajustamento. Todos os coeficientes são estatisticamente significativos na relação de longo prazo. Na relação de curto prazo apenas os coeficientes do câmbio, IPCA e M1 são estatisticamente significativos.

Tabela 2 - Coeficiente de cointegração normalizado

\begin{tabular}{cccccccc}
\hline LPIB & LDIVIDAPIB & LSP & LCAMBIO & IPCA1 & LEXPIPCA & LM1 & LSELIC \\
\hline $\mathbf{1}$ & $\mathbf{1 , 9 8 8 5}$ & $\mathbf{0 , 2 7 2 9}$ & $\mathbf{- 0 , 7 3 6 9}$ & $\mathbf{0 , 1 1 5 3}$ & $\mathbf{- 1 , 7 4 1 0}$ & $\mathbf{- 4 , 4 0 6 2}$ & $\mathbf{- 0 , 7 3 2 3}$ \\
& $(0,6411)$ & $(0,0972)$ & $(0,3458)$ & $(0,0143)$ & $(0,3311)$ & $(0,9115)$ & $(0,2849)$ \\
\hline
\end{tabular}

Nota: Desvio padrão entre parênteses.

Fonte: Resultados da pesquisa. 
As conclusões que se podem extrair desses relacionamentos de longo prazo mostram algumas indicações de possíveis respostas para as questões colocadas acima, especialmente aquelas relacionadas ao impacto de longo prazo de algumas variáveis de política monetária e fiscal no crescimento econômico. A equação (7) apresenta a relação de longo prazo normalizado para o PIB brasileiro.

$$
\begin{aligned}
& L P I B_{t}= \\
& \beta_{0}-1,99 \text { Ldivpib }_{t}-0,27 L S P_{t}+0,74 \text { Lcâmbio }_{t}-0,12 \text { Lipca }_{t}+1,74 \text { Lexpipca }_{t}+ \\
& 4,41 L m 1_{t}+0,73 L \text { Selic }_{t}+\varepsilon_{t}
\end{aligned}
$$

O coeficiente da variável dívida pública indica que dado um aumento de $1 \%$ na dívida, o PIB brasileiro pode decrescer quase $2 \%$, impacto significativo na relação de longo prazo. Mesmo com algum declínio nos últimos anos, a dívida pública brasileira ainda é bastante representativa quando medida em termos do PIB. Mais de 50\% da dívida pública do Brasil está indexada à taxa de juros, que é definida pela autoridade de política monetária. Nesse sentido, aumento da taxa de juros impacta fortemente na dívida pública.

O modelo apresentou uma relação inversa entre o PIB e o superavit primário. O coeficiente do superavit primário sugere que um aumento de $1 \%$ pode acarretar declínio no PIB na ordem de 0,27\%. Alguns autores acreditam que a redução do superavit permite ao governo expandir seus investimentos, gerando um impacto expansionista sobre o produto. Com isso a relação dívida/PIB cairia, o que possibilitaria queda dos juros reais, o que, por sua vez, estimularia mais ainda a expansão do produto. O superavit primário é a economia de recursos para pagar os juros da dívida pública e reduzir o endividamento do governo no médio e no longo prazos.

Em relação ao câmbio, a equação (7) sugere que variações positivas nessa variável contribuem para variações positivas no PIB do Brasil. Note que variação positiva no câmbio se trata de desvalorização da moeda brasileira, o que pode influenciar as exportações, já que o produto brasileiro se torna mais competitivo no mercado externo. Quanto ao coeficiente da oferta de moeda, se esta sofrer alteração de $1 \%$, haverá crescimento de cerca de mais de $4 \%$ do PIB. A variável de oferta de moeda induz uma variação mais significativa no PIB. Seguindo a teoria de crescimento econômico, o IPCA afeta negativamente as variações do PIB, sugerindo que a cada variação de $1 \%$ da inflação, o PIB varia negativamente em $0,12 \%$.

Já a expectativa é a de a inflação estar com sinal errado e coeficiente alto, embora significativo estatisticamente. O mesmo aconteceu com a taxa de juros. De acordo com a literatura, elevações na taxa de juros tendem a inibir a demanda agregada e aumentar o fluxo de capitais, com consequente apreciação cambial, induzindo a diminuições no PIB interno, e aumentando as importações. Blanchard (2004) sugere que no caso da economia brasileira, uma alta taxa de juros tende a elevar a razão dívida/PIB. 
Como o endividamento é significativo, esse crescimento pode aumentar a percepção de riscos dos agentes e, consequentemente, a probabilidade de default.

A velocidade do coeficiente de ajuste pode ser conferida na Tabela 3. Após desequilíbrio de curto prazo no PIB, os coeficientes de correção de erros mostram que o ajuste médio é muito baixo $(0,003)$ na equação de cointegração. Portanto, esse ajuste mostra uma pequena tendência de melhora do crescimento do PIB brasileiro para o desequilíbrio em curto prazo, sendo a outra parte corrigida pelas relações de longo prazo. A taxa de inflação é corrigida com maior velocidade quando sai da sua trajetória de longo prazo, conforme o valor do coeficiente de ajustamento.

Tabela 3 - Coeficientes de ajustamento

\begin{tabular}{cccccccc}
\hline \multirow{2}{*}{$\mathrm{D}(\mathrm{LPIB})$} & $\mathrm{D}(\mathrm{LDIVIDA}$ & $\mathrm{D}$ & $\mathrm{D}$ & $\mathrm{D}$ & $\mathrm{D}(\mathrm{LEXP}$ & $\mathrm{D}$ & $\mathrm{D}$ \\
& $\mathrm{PIB})$ & $(\mathrm{LSP})$ & $($ LCAMBIO) & $(\mathrm{IPCA} 1)$ & IPCA $)$ & $(\mathrm{LM} 1)$ & $($ LSELIC) \\
\hline $\mathbf{0 , 0 0}$ & $\mathbf{0 , 0 0}$ & $\mathbf{- 0 , 3 0}$ & $\mathbf{0 , 0 1}$ & $\mathbf{- 5 , 2 5}$ & $\mathbf{0 , 0 0}$ & $\mathbf{- 0 , 0 2}$ & $\mathbf{0 , 0 2}$ \\
$(0,00)$ & $(0,00)$ & $(0,07)$ & $(0,01)$ & $(0,89)$ & $(0,01)$ & $(0,01)$ & $(0,02)$ \\
\hline
\end{tabular}

Nota: Desvio padrão entre parênteses.

Fonte: Resultados da pesquisa.

Quanto aos testes dos resíduos, há evidência de ausência de correlação serial nos resíduos a $1 \%$ de significância, mas se rejeita a hipótese de normalidade para os resíduos do modelo, embora testes para resíduos de algumas equações individuais não rejeitem normalidade. Também foi possível rejeitar a hipótese de ausência de heteroscedasticidade condicional. Como a não normalidade não é um problema grave, o modelo é aproximadamente congruente.

Na Tabela 4 é apresentada a análise impulso resposta apenas para o PIB.

Tabela 4 - Análise impulso resposta do PIB

\begin{tabular}{ccccccccc}
\hline Período & LPIB & LDIVIDAPIB & LSP & LCAMBIO & IPCA1 & LEXPIPCA & LM1 & LSELIC \\
\hline 1 & 0,0281 & 0,0000 & 0,0000 & 0,0000 & 0,0000 & 0,0000 & 0,0000 & 0,0000 \\
3 & 0,0259 & 0,0025 & 0,0012 & $-0,0056$ & $-0,0032$ & 0,0035 & $-0,0181$ & $-0,0115$ \\
6 & 0,0214 & 0,0047 & 0,0025 & $-0,0101$ & 0,0036 & $-0,0002$ & $-0,0109$ & $-0,0063$ \\
9 & 0,0199 & 0,0076 & 0,0038 & $-0,008$ & 0,0017 & 0,0012 & $-0,0102$ & $-0,0071$ \\
12 & 0,0205 & 0,0066 & 0,0034 & $-0,0079$ & 0,0020 & 0,0005 & $-0,0103$ & $-0,0077$ \\
\hline
\end{tabular}

Fonte: Resultados da pesquisa.

O M1 se comporta de modo previsto pela teoria de política monetária, sendo que a demanda por moeda tende a reduzir a um aumento no custo de oportunidade ao se reterem meios de pagamentos líquidos, com um efeito máximo de $1 \%$ em 12 meses. A resposta à taxa de câmbio atinge seu ponto máximo (0,10 p.p.) em aproximadamente seis meses, e o efeito cessa em pouco menos de dois anos. As variáveis de política monetária 
respondem ao impulso negativamente. Esse resultado indica, entre outras coisas, que a política monetária, sob o regime de metas inflacionárias, tem obtido sucesso não apenas em afetar negativamente a produção por meio de um choque exógeno, mas também em criar uma reputação em que a sinalização futura, feita por meio de suas medidas e de seus pronunciamentos, tem efeitos reais sobre a economia. A resposta ao impulso do superavit primário aumenta a partir do $1^{\circ}$ período e recua um pouco a partir do $12^{\circ}$ mês.

Na Tabela 5 é apresentada a análise da decomposição da variância também para o PIB.

Tabela 5 - Análise decomposição da variância do PIB

\begin{tabular}{ccccccccc}
\hline Período & LPIB & LDIVIDAPIB & LSP & LCAMBIO & IPCA1 & LEXPIPCA & LM1 & LSELIC \\
\hline 1 & 100,0000 & 0,0000 & 0,0000 & 0,0000 & 0,0000 & 0,0000 & 0,0000 & 0,0000 \\
3 & 76,7666 & 0,3316 & 0,1069 & 1,1207 & 0,5540 & 0,4434 & 13,1078 & 7,5686 \\
6 & 68,3846 & 1,58228 & 0,9650 & 6,8614 & 0,6453 & 0,4563 & 14,4832 & 6,6215 \\
9 & 65,0208 & 3,3608 & 1,1383 & 7,2884 & 0,7412 & 0,3810 & 14,6986 & 7,3705 \\
12 & 63,4899 & 4,0853 & 1,27151 & 7,8499 & 0,6975 & 0,2956 & 14,7920 & 7,5181 \\
\hline
\end{tabular}

Fonte: Resultados da pesquisa.

A variância do crescimento econômico do país indica ter sofrido influência significativa das variáveis. As variações do produto em relação à taxa de juros foram de aproximadamente 7\%, advindas de choques pela tomada de decisão de política monetária ao definir a taxa de juros. Observa-se que os choques da variável M1 são mais fortes sobre o PIB, o que pode ser traduzido como uma preocupação inicial das autoridades monetárias com o crescimento econômico do país. A influência da dívida pública na composição da variância do PIB chega a 4\% em 12 meses, o que pode ser preocupante, dado que esse valor cresce gradativamente.

Em suma, pelos motivos já expostos, acredita-se que a análise isolada tanto da política fiscal quanto da política monetária não é suficiente para medir os impactos dessas políticas sobre a economia, sobretudo quando analisadas as variações do PIB. É importante novamente frisar que a interação conjunta da política monetária e da política fiscal é fundamental para tentar entender as variações do PIB quando tomadas as decisões de políticas econômicas por parte das autoridades.

\section{CONSIDERAÇÕES FINAIS}

Esse artigo teve como objetivo verificar empiricamente a relação dinâmica das variáveis de política monetária e política fiscal com o crescimento econômico brasileiro no período recente, após a implantação do sistema de metas de inflação, por meio da 
modelagem VEC. Desenvolvimentos recentes, tanto empíricos quanto teóricos, têm evidenciado crescente ligação entre a política monetária e fiscal na explicação de desequilíbrios macroeconômicos.

A principal contribuição deste artigo foi buscar uma interpretação econométrica estrutural das variáveis de políticas fiscal e monetária com o crescimento econômico do país. Os resultados corroboraram a literatura que trata da existência de uma relação negativa entre crescimento e inflação. Dessa forma é importante que as autoridades de política monetária possam buscar sempre a estabilidade dos preços na economia e contribuir com o crescimento econômico. Do lado da política fiscal, os resultados mostraram que é necessário um controle intensivo das contas públicas, dada a relação negativa da dívida pública com o crescimento econômico. Além disso, a política cambial também se mostrou necessária nesse processo, uma vez que o câmbio pode ser uma ferramenta importante para alavancar as exportações e aumentar a competitividade do país.

Na relação de curto prazo, o coeficiente de ajuste foi positivo para os possíveis desequilíbrios do PIB. Embora o coeficiente seja pequeno, mostra uma tendência de melhora no crescimento econômico no curto prazo.

Esses resultados são interessantes no sentido de que vários trabalhos na literatura têm mostrado a importância da coordenação das autoridades de políticas econômicas com o crescimento econômico, principalmente em um arcabouço de metas de inflação.

\section{REFERÊNCIAS}

AMARAL, R. Q.; OREIRO, J. L. A relação entre o mercado de dívida pública e a política monetária no Brasil. Revista de Economia Contemporânea, v. 12, n. 3, p. 491-517, 2008.

BARRO, R. J. Government spending in simple model of endogenous growth. Journal of Political Economy, v. 98, n. 5, p. 103-125, 1990.

BENIGNO, P.; WOODFORD, M. Optimal stabilization policy when wages and prices are sticky: the case of a distorted steady state. USA: Board of Governors of the Federal Reserve System, p. 127-180, 2004.

BLANCHARD, O. Fiscal dominance and inflation targeting: lessons from Brazil. Working Paper, n. 10.389, 2004.

BUENO, R. L. S. Econometria de séries temporais. São Paulo: Cengage Learning, 2008.

CHARI, V. V.; JONES, L. E.; MANUELLI, R. E. The growth effects of monetary policy. Federal Reserve Bank of Minneapolis Quartely Review, v. 19, n. 4, p. 18-32, 1995.

COCHRANE, J. H. A frictionless view of the US inflation. NBER Working Paper Series, n. 6646, 1998. 
COCHRANE, J. H. Long term debt and optimal policy in the fiscal theory of the price level. Econometrica, v. 69, n. 1, 2001.

ENGLE, R. F.; GRANGER, C. W. J. Co-integration and error correction: representation, estimation and testing. Econometrica, v. 55, n. 2, p. 251-276, 1987.

FAVERO, C. A. Comments on "Fiscal and monetary policy interactions: empirical evidence on optimal policy using a structural new-keynesian model". Journal of Macroeconomics, v. 26, n. 2, p. 281-285, 2004.

JOHANSEN, S. J. Likelihood-based inference in cointegrated vector autoregressive models, advanced texts in econometrics. Oxford: Oxford University Press, 1996.

JUNIOR, K. M.; JESUS, C. S. A condução da política fiscal numa economia aberta com metas de inflação. A Economia em Revista, v. 18, n. 2, p. 37-47, 2010.

KING, R. G.; REBELO, S. Public Policy and Economic Growth: developing neoclassical implications. NBER Working Paper Series, n. 3338, 1990.

KYDLAND, F. E.; PRESCOTT, E. C. Rules rather than discretion: the inconsistency of optimal plans. Journal of Political Economy, p. 473-91, 1977.

LEEPER, E. M. Equilibria under "active" and "passive" monetary and fiscal policies. Journal of Monetary Economics, v. 27, n. 1, p. 129-147, 1991.

LUCAS, R. E. Jr. Econometric policy evaluation: a critique. Studies in Business-Cycle Theory, MIT Press, 1983.

LUCAS, R. E. Jr. On the mechanics of economic development. Journal of Monetary Economics, v. 22, n. 1, p. 3-42, 1988.

MENDONÇA, H. F. "Coordenação de políticas macroeconômicas: implicações para o caso brasileiro”. In: BRASIL. Ministério da Fazenda. Secretaria do Tesouro Nacional. Finanças públicas: V Prêmio Tesouro Nacional; Coletânea de Monografia. Brasília: ESAF, 2001, p. 165241.

MUSA, Y.; ASARE, B. K. Long and short run relationship analysis of monetary and fiscal policy on economic growth in Nigeria: a VEC model approach. Journal of Applied Sciences, Engineering and Technology, v. 5, n. 10, p. 3044-3051, 2013.

PASTORE, A. C. Por que a política monetária perde eficácia? Revista Brasileira de Economia, v. 50, p. 281-311, 1995.

PEROTTI, R. Estimating the effects of fiscal policy in OECD countries. Technical report. Working Paper, European Central Bank, n. 168, 2002.

PERSSON, M.; PERSSON, T.; SVENSSON, L. E. O. Time consistency of fiscal and monetary policy: a solution. New Jersey: Institute for International Economic Studies \& Princeton University, 2004. Mimeo.

PLOEG, F. V. D.; ALOGOSKOUFIS, G. S. Money and Endogenous Growth. Journal of Money, Credit and Banking, v. 26, n. 4. 1994.

REBELO, S. Long-run policy analysis and long-run growth . Journal of Political Economy, v. 99, n. 3, p. 500-521, 1991.

ROCHA, F. F.; PASCHOALOTTO, E. Teoria fiscal do nível de preços: um teste para a economia brasileira no período 1966/2000. Pesquisa e Planejamento Econômico, v. 34, n. 3, 2004. 
ROMER, P. M. Increasing returns and long-run growth. Journal of Political Economy, v. 94, n. 5, p. 1002-1037, 1986.

SARGENT, T. J. Beyond demand and supply curves in Macroeconomics. The American Economic Review, v. 72, n. 2, 1982.

SARGENT, T. J.; WALLACE, N. Some unpleasant monetarist arithmetic. Federal Reserve Bank of Minneapolis Quarterly Review, v. 5, n. 3, p. 1-17, 1981.

SCHMITT-GROHE, S.; URIBE, M. Optimal fiscal and monetary policy under imperfect competition. Journal of Macroeconomics, v. 26, p. 183-209, 2004a.

SCHMITT-GROHE, S.; URIBE, M. Optimal fiscal and monetary policy under sticky prices. Journal of Economic Theory, v. 114, n. 2, p. 198-230, 2004 b.

SCHMITT-GROHE, S.; URIBE, M. "Optimal fiscal and monetary policy in a medium scale macroeconomic model”. In: GERTLER, M.; ROGOFF, K. (Eds.). NBER Macroeconomics Annual 2005. Cambridge: The MIT Press, 2006, p. 383-425.

SIMS, C. A. A simple model for study of the price level and the interaction of monetary and fiscal policy. Economic Theory, v. 4, n. 3, p. 381-399, 1994.

SIMS, C. A. Limits to inflation targeting. Technical report. Working Paper, Princeton University, Department of Economics, 2003.

TAYLOR, J. B. Discretion versus policy rules in practice. Carnegie- Rochester Conference Series on Public Policy, v. 39, 1993.

WOODFORD, M. Monetary policy and price-level determinacy in a cash-in-advance economy. Econometric Theory, v. 4, n. 3, p. 345-380, 1994.

WOODFORD, M. Price level determinacy without control of a monetary aggregate. NBER Working Paper Series, n. 5204, 1995.

WOODFORD, M. Fiscal requirements for price stability. Journal of Money, Credit and Banking, v. 33, p. 669-728, 2001. 


\section{Anexo - Séries históricas utilizadas no modelo}

Tabela A-1 - Variáveis utilizadas no modelo

\begin{tabular}{|c|c|c|c|c|c|c|c|c|}
\hline PERÍODO & PIB & DIVIDAPIB & SP & CAMBIO & IPCA & EXPIPCA & M1 & SELIC \\
\hline jul/01 & 108944,20 & 49,47 & 1,17 & 2,47 & 17,18 & 6,40 & 5,07 & 1,50 \\
\hline ago/01 & 110244,10 & 49,97 & 1,36 & 2,51 & 8,73 & 6,57 & 5,06 & 1,60 \\
\hline set/01 & 105062,60 & 50,47 & 1,29 & 2,67 & 3,41 & 6,53 & 5,09 & 1,32 \\
\hline out/01 & 114176,90 & 50,98 & 1,09 & 2,74 & 10,43 & 6,49 & 4,97 & 1,53 \\
\hline nov/01 & 117611,70 & 51,50 & 1,36 & 2,54 & 8,86 & 5,57 & 5,15 & 1,39 \\
\hline $\mathrm{dez} / 01$ & 114429,80 & 52,02 & 1,94 & 2,36 & 8,08 & 4,84 & 6,14 & 1,39 \\
\hline $\mathrm{jan} / 02$ & 112179,60 & 52,80 & 1,54 & 2,38 & 6,42 & 4,76 & 5,46 & 1,53 \\
\hline $\mathrm{fev} / 02$ & 110052,20 & 51,93 & 1,74 & 2,42 & 4,41 & 4,70 & 5,40 & 1,25 \\
\hline $\operatorname{mar} / 02$ & 115637,00 & 51,50 & 2,13 & 2,35 & 7,44 & 4,94 & 5,32 & 1,37 \\
\hline $\mathrm{abr} / 02$ & 120299,40 & 51,42 & 2,16 & 2,32 & 10,03 & 4,90 & 5,37 & 1,48 \\
\hline $\mathrm{mai} / 02$ & 124666,70 & 52,61 & 2,61 & 2,48 & 2,55 & 4,37 & 5,22 & 1,41 \\
\hline jun/02 & 125751,00 & 54,94 & 2,16 & 2,71 & 5,16 & 4,57 & 5,49 & 1,33 \\
\hline $\mathrm{jul} / 02$ & 125668,30 & 59,19 & 1,38 & 2,93 & 15,25 & 5,08 & 5,56 & 1,54 \\
\hline ago/02 & 125228,80 & 56,27 & 1,90 & 3,11 & 8,08 & 5,40 & 5,60 & 1,44 \\
\hline set/02 & 121289,10 & 62,86 & 1,33 & 3,34 & 8,99 & 6,28 & 5,58 & 1,38 \\
\hline out/02 & 130929,20 & 60,80 & 1,57 & 3,81 & 16,90 & 8,94 & 5,36 & 1,65 \\
\hline nov/02 & 135194,60 & 60,26 & 1,19 & 3,58 & 42,91 & 12,47 & 5,35 & 1,54 \\
\hline $\mathrm{dez} / 02$ & 130926,20 & 60,38 & 0,68 & 3,63 & 28,32 & 13,24 & 6,18 & 1,74 \\
\hline $\mathrm{jan} / 03$ & 129392,70 & 60,26 & 1,11 & 3,44 & 30,60 & 11,72 & 5,24 & 1,97 \\
\hline fev/03 & 128202,00 & 60,61 & 1,02 & 3,59 & 20,56 & 11,07 & 5,12 & 1,83 \\
\hline $\mathrm{mar} / 03$ & 133986,30 & 58,93 & 1,12 & 3,45 & 15,80 & 9,90 & 4,91 & 1,78 \\
\hline abr/03 & 137600,30 & 54,99 & 0,71 & 3,12 & 12,28 & 9,18 & 4,83 & 1,87 \\
\hline $\mathrm{mai} / 03$ & 142330,30 & 55,47 & 0,97 & 2,96 & 7,57 & 8,23 & 4,85 & 1,97 \\
\hline jun/03 & 140303,70 & 54,92 & 1,57 & 2,88 & $-1,79$ & 7,08 & 5,06 & 1,86 \\
\hline $\mathrm{jul} / 03$ & 141286.80 & 55,68 & 2,69 & 2,88 & 2,43 & 6,39 & 5,02 & 2,08 \\
\hline ago/03 & 142502,40 & 56,03 & 2,68 & 3,00 & 4,16 & 6,32 & 5,01 & 1,77 \\
\hline set/03 & 146086,20 & 55,27 & 3,42 & 2,92 & 9,77 & 6,56 & 5,03 & 1,68 \\
\hline out/03 & 152695,60 & 54,43 & 3,00 & 2,86 & 3,54 & 6,15 & 5,01 & 1,64 \\
\hline nov/03 & 152652,60 & 54,89 & 3,29 & 2,91 & 4,16 & 5,80 & 5,36 & 1,34 \\
\hline $\mathrm{dez} / 03$ & 152908,90 & 54,83 & 3,66 & 2,93 & 6,42 & 5,92 & 6,29 & 1,37 \\
\hline jan/04 & 147296,40 & 54,58 & 3,37 & 2,85 & 9,51 & 6,01 & 5,53 & 1,27 \\
\hline $\mathrm{fev} / 04$ & 140745,50 & 54,26 & 3,28 & 2,93 & 7,57 & 5,74 & 5,53 & 1,08 \\
\hline $\mathrm{mar} / 04$ & 151581,50 & 53,72 & 2,99 & 2,91 & 5,79 & 5,54 & 5,34 & 1,38 \\
\hline abr/04 & 153068,90 & 53,51 & 3,24 & 2,91 & 4,53 & 5,60 & 5,25 & 1,18 \\
\hline $\mathrm{mai} / 04$ & 163284.00 & 54,02 & 2,87 & 3,10 & 6,29 & 5,98 & 5,33 & 1,23 \\
\hline jun/04 & 167458,30 & 53,31 & 2,41 & 3,13 & 8,86 & 6,33 & 5,29 & 1,23 \\
\hline $\mathrm{jul} / 04$ & 164556,00 & 52,55 & 1,95 & 3,04 & 11,48 & 6,36 & 5,25 & 1,29 \\
\hline ago/04 & 166360,90 & 51,91 & 1,67 & 3,00 & 8,60 & 6,27 & 5,32 & 1,29 \\
\hline set/04 & 164494,60 & 51,32 & 1,05 & 2,89 & 4,03 & 6,24 & 5,50 & 1,25 \\
\hline out/04 & 171571,70 & 51,13 & 1,61 & 2,85 & 5,41 & 6,22 & 5,46 & 1,21 \\
\hline nov/04 & 175391,60 & 50,44 & 1,58 & 2,79 & 8,60 & 6,30 & 5,60 & 1,25 \\
\hline $\mathrm{dez} / 04$ & 175688,50 & 50,61 & 1,39 & 2,72 & 10,82 & 6,08 & 6,28 & 1,48 \\
\hline jan/05 & 167348,50 & 49,95 & 1,41 & 2,69 & 7,19 & 5,74 & 5,81 & 1,38 \\
\hline $\mathrm{fev} / 05$ & 157972,20 & 49,71 & 1,63 & 2,60 & 7,31 & 5,61 & 5,72 & 1,22 \\
\hline
\end{tabular}


Tabela A-1 - Variáveis utilizadas no modelo (continuação)

\begin{tabular}{|c|c|c|c|c|c|c|c|c|}
\hline PERÍODO & PIB & DIVIDAPIB & SP & CAMBIO & IPCA & EXPIPCA & M1 & SELIC \\
\hline $\operatorname{mar} / 05$ & 168842,20 & 49,72 & 1,76 & 2,70 & 7,57 & 5,67 & 5,64 & 1,53 \\
\hline $\mathrm{abr} / 05$ & 174036,40 & 48,67 & 1,55 & 2,58 & 10,95 & 5,87 & 5,47 & 1,41 \\
\hline mai/05 & 178714,30 & 48,34 & 1,95 & 2,45 & 6,04 & 5,46 & 5,59 & 1,50 \\
\hline jun/05 & 181814,80 & 48,50 & 2,32 & 2,41 & $-0,24$ & 4,85 & 5,66 & 1,59 \\
\hline jul/05 & 177507,10 & 48,61 & 2,45 & 2,37 & 3,04 & 4,86 & 5,66 & 1,51 \\
\hline ago/05 & 183406,00 & 48,46 & 2,62 & 2,36 & 2,06 & 4,70 & 5,66 & 1,66 \\
\hline set/05 & 181803,90 & 48,11 & 3,12 & 2,29 & 4,28 & 4,74 & 5,62 & 1,50 \\
\hline out $/ 05$ & 188842,50 & 48,01 & 3,16 & 2,26 & 9,38 & 4,73 & 5,66 & 1,41 \\
\hline nov/05 & 193858,40 & 47,89 & 3,35 & 2,21 & 6,80 & 4,61 & 5,93 & 1,38 \\
\hline $\mathrm{dez} / 05$ & 193092,60 & 48,44 & 3,41 & 2,29 & 4,41 & 4,46 & 6,71 & 1,47 \\
\hline $\mathrm{jan} / 06$ & 182558,40 & 48,45 & 3,93 & 2,27 & 7,31 & 4,62 & 5,97 & 1,43 \\
\hline $\mathrm{fev} / 06$ & 176778,50 & 48,36 & 3,92 & 2,16 & 5,03 & 4,40 & 6,00 & 1,15 \\
\hline $\mathrm{mar} / 06$ & 186407,10 & 48,23 & 4,01 & 2,15 & 5,28 & 4,31 & 5,86 & 1,42 \\
\hline abr/06 & 182323,00 & 47,76 & 4,08 & 2,13 & 2,55 & 4,06 & 5,77 & 1,08 \\
\hline mai/06 & 196432,90 & 47,69 & 3,70 & 2,18 & 1,21 & 4,10 & 5,85 & 1,28 \\
\hline jun/06 & 198625,50 & 47,59 & 3,67 & 2,25 & $-2,49$ & 3,98 & 5,85 & 1,18 \\
\hline jul/06 & 199648,20 & 47,45 & 3,64 & 2,19 & 2,30 & 4,28 & 5,86 & 1,17 \\
\hline ago/06 & 205321,30 & 47,22 & 3,53 & 2,16 & 0,60 & 4,40 & 5,93 & 1,26 \\
\hline set/06 & 198747,80 & 47,26 & 3,30 & 2,17 & 2,55 & 3,93 & 6,16 & 1,06 \\
\hline out/06 & 210072,60 & 46,94 & 3,19 & 2,15 & 4,03 & 4,00 & 6,18 & 1,09 \\
\hline nov/06 & 217897,70 & 46,78 & 3,10 & 2,16 & 3,78 & 4,14 & 6,41 & 1,02 \\
\hline $\mathrm{dez} / 06$ & 214671,10 & 47,27 & 3,14 & 2,15 & 5,91 & 4,08 & 7,30 & 0,99 \\
\hline jan/07 & 207646,30 & 46,71 & 2,54 & 2,14 & 5,41 & 4,03 & 6,35 & 1,08 \\
\hline fev/07 & 198028,40 & 46,68 & 2,45 & 2,10 & 5,41 & 3,81 & 6,23 & 0,87 \\
\hline $\mathrm{mar} / 07$ & 212139,80 & 46,66 & 2,53 & 2,09 & 4,53 & 3,74 & 6,27 & 1,05 \\
\hline abr/07 & 211806,70 & 45,92 & 2,47 & 2,03 & 3,04 & 3,47 & 6,26 & 0,94 \\
\hline mai/07 & 225232,40 & 46,06 & 2,79 & 1,98 & 3,41 & 3,40 & 6,27 & 1,03 \\
\hline jun/07 & 224942,30 & 45,67 & 2,56 & 1,93 & 3,41 & 3,47 & 6,46 & 0,91 \\
\hline jul/07 & 222545,90 & 45,67 & 2,57 & 1,88 & 2,92 & 3,55 & 6,49 & 0,97 \\
\hline ago/07 & 229310,20 & 45,03 & 2,47 & 1,97 & 5,79 & 3,83 & 6,51 & 0,99 \\
\hline set/07 & 221509,30 & 45,50 & 2,50 & 1,90 & 2,18 & 3,75 & 6,62 & 0,80 \\
\hline out/07 & 236445,90 & 45,52 & 2,40 & 1,80 & 3,66 & 3,71 & 6,66 & 0,93 \\
\hline nov/07 & 238323,30 & 45,04 & 2,17 & 1,77 & 4,66 & 3,85 & 6,94 & 0,84 \\
\hline $\mathrm{dez} / 07$ & 233413.50 & 45,53 & 2,24 & 1,79 & 9,25 & 4,45 & 8,25 & 0,84 \\
\hline jan/08 & 234345,60 & 44,66 & 1,86 & 1,77 & 6,68 & 4,44 & 6,72 & 0,93 \\
\hline $\mathrm{fev} / 08$ & 226356,90 & 44,77 & 1,89 & 1,73 & 6,04 & 4,28 & 6,44 & 0,80 \\
\hline $\mathrm{mar} / 08$ & 233673,20 & 43,99 & 1,46 & 1,71 & 5,91 & 4,36 & 6,38 & 0,84 \\
\hline abr/08 & 242222,00 & 43,85 & 1,37 & 1,69 & 6,80 & 4,56 & 6,37 & 0,90 \\
\hline mai/08 & 254822,70 & 44,03 & 1,20 & 1,66 & 9,90 & 5,08 & 6,14 & 0,88 \\
\hline jun/08 & 261466,80 & 43,88 & 1,13 & 1,62 & 9,25 & 5,60 & 6,14 & 0,96 \\
\hline jul/08 & 265042,30 & 43,68 & 0,95 & 1,59 & 6,55 & 5,49 & 6,09 & 1,07 \\
\hline ago/08 & 262570,00 & 42,96 & 0,74 & 1,61 & 3,41 & 5,16 & 6,11 & 1,02 \\
\hline set/08 & 260078,50 & 40,93 & 0,40 & 1,80 & 3,17 & 5,01 & 6,31 & 1,10 \\
\hline out/08 & 276639,10 & 39,21 & 0,00 & 2,17 & 5,54 & 5,40 & 6,10 & 1,18 \\
\hline nov/08 & 268166,00 & 37,81 & 0,19 & 2,27 & 4,41 & 5,45 & 6,37 & 1,02 \\
\hline $\mathrm{dez} / 08$ & 246819,70 & 38,53 & 0,82 & 2,39 & 3,41 & 4,93 & 7,23 & 1,12 \\
\hline jan/09 & 243770,70 & 39,06 & 1,47 & 2,31 & 5,91 & 4,70 & 6,45 & 1,05 \\
\hline
\end{tabular}


Tabela A-1 - Variáveis utilizadas no modelo (continuação)

\begin{tabular}{|c|c|c|c|c|c|c|c|c|}
\hline PERÍODO & PIB & DIVIDAPIB & SP & CAMBIO & IPCA & EXPIPCA & M1 & SELIC \\
\hline fev/09 & 234953,60 & 39,05 & 1,49 & 2,31 & 6,80 & 4,57 & 6,39 & 0,86 \\
\hline $\mathrm{mar} / 09$ & 250676,00 & 39,11 & 1,82 & 2,31 & 2,43 & 4,14 & 6,29 & 0,97 \\
\hline abr/09 & 253179,10 & 39,73 & 2,13 & 2,21 & 5,91 & 4,21 & 6,35 & 0,84 \\
\hline mai/09 & 264321,30 & 40,81 & 2,31 & 2,06 & 5,79 & 4,14 & 6,38 & 0,77 \\
\hline jun/09 & 270462,30 & 41,15 & 2,65 & 1,96 & 4,41 & 4,06 & 6,58 & 0,76 \\
\hline jul/09 & 270895,00 & 41,89 & 3,00 & 1,93 & 2,92 & 4,09 & 6,45 & 0,79 \\
\hline ago/09 & 277382,40 & 41,87 & 3,33 & 1,85 & 1,81 & 3,97 & 6,57 & 0,69 \\
\hline set/09 & 278153,70 & 42,79 & 4,06 & 1,82 & 2,92 & 4,19 & 6,77 & 0,69 \\
\hline out/09 & 284249,90 & 42,86 & 4,40 & 1,74 & 3,41 & 4,32 & 6,77 & 0,69 \\
\hline nov/09 & 298001,60 & 42,42 & 4,17 & 1,73 & 5,03 & 4,35 & 7,06 & 0,66 \\
\hline $\mathrm{dez} / 09$ & 313358,40 & 42,07 & 3,31 & 1,75 & 4,53 & 4,40 & 7,86 & 0,73 \\
\hline $\mathrm{jan} / 10$ & 283098,70 & 40,72 & 2,93 & 1,78 & 9,38 & 4,68 & 6,94 & 0,66 \\
\hline $\mathrm{fev} / 10$ & 276565,40 & 40,93 & 2,95 & 1,84 & 9,77 & 4,66 & 6,78 & 0,59 \\
\hline $\mathrm{mar} / 10$ & 295904,60 & 41,07 & 3,12 & 1,79 & 6,42 & 4,70 & 6,81 & 0,76 \\
\hline $\mathrm{abr} / 10$ & 297345,50 & 40,65 & 2,79 & 1,76 & 7,06 & 4,86 & 6,71 & 0,67 \\
\hline $\mathrm{mai} / 10$ & 312832,40 & 40,11 & 2,77 & 1,81 & 5,28 & 4,88 & 6,69 & 0,75 \\
\hline jun/10 & 316919,10 & 39,99 & 2,58 & 1,81 & 0,00 & 4,74 & 6,70 & 0,79 \\
\hline $\mathrm{jul} / 10$ & 318291,00 & 40,08 & 2,53 & 1,77 & 0,12 & 4,82 & 6,64 & 0,86 \\
\hline ago/10 & 323221,20 & 39,87 & 2,50 & 1,76 & 0,48 & 4,98 & 6,75 & 0,89 \\
\hline set/10 & 321926,10 & 39,35 & 1,41 & 1,72 & 5,54 & 5,31 & 6,83 & 0,85 \\
\hline out/10 & 336583,80 & 38,88 & 1,43 & 1,68 & 9,38 & 5,42 & 6,76 & 0,81 \\
\hline nov/10 & 345097,10 & 38,78 & 1,62 & 1,71 & 10,43 & 5,61 & 6,93 & 0,81 \\
\hline $\mathrm{dez} / 10$ & 342299.80 & 39,15 & 1,21 & 1,69 & 7,83 & 5,52 & 7,48 & 0,91 \\
\hline $\mathrm{jan} / 11$ & 321447,20 & 38,76 & 1,28 & 1,67 & 10,43 & 5,67 & 6,76 & 0,86 \\
\hline $\mathrm{fev} / 11$ & 315854,60 & 38,76 & 1,29 & 1,67 & 10,03 & 5,61 & 6,61 & 0,84 \\
\hline $\operatorname{mar} / 11$ & 324770,80 & 38,88 & 1,01 & 1,66 & 9,90 & 5,61 & 6,50 & 0,92 \\
\hline abr/11 & 331041,90 & 38,83 & 1,16 & 1,59 & 9,64 & 4,86 & 6,35 & 0,84 \\
\hline $\mathrm{mai} / 11$ & 354476,90 & 38,76 & 1,16 & 1,61 & 5,79 & 4,88 & 6,32 & 0,99 \\
\hline jun/11 & 358007,80 & 38,62 & 1,15 & 1,59 & 1,81 & 4,74 & 6,36 & 0,96 \\
\hline $\mathrm{jul} / 11$ & 347660,20 & 38,42 & 0,98 & 1,56 & 1,94 & 5,35 & 6,28 & 0,97 \\
\hline ago/11 & 355029,80 & 38,22 & 1,17 & 1,60 & 4,53 & 5,47 & 6,13 & 1,07 \\
\hline set/11 & 344016,40 & 36,34 & 1,69 & 1,75 & 6,55 & 5,80 & 6,26 & 0,94 \\
\hline out/11 & 360556,60 & 37,44 & 1,72 & 1,77 & 5,28 & 5,56 & 6,17 & 0,88 \\
\hline nov/11 & 366016,80 & 36,60 & 1,68 & 1,79 & 6,42 & 5,53 & 6,34 & 0,86 \\
\hline $\mathrm{dez} / 11$ & 364134,10 & 36,41 & 2,11 & 1,84 & 6,17 & 5,35 & 6,89 & 0,91 \\
\hline $\mathrm{jan} / 12$ & 337814,30 & 37,18 & 2,03 & 1,79 & 6,93 & 5,30 & 6,25 & 0,89 \\
\hline $\mathrm{fev} / 12$ & 333895,40 & 37,57 & 2,00 & 1,72 & 5,54 & 5,26 & 6,15 & 0,75 \\
\hline $\mathrm{mar} / 12$ & 352629,10 & 36,50 & 2,14 & 1,80 & 2,55 & 5,43 & 6,12 & 0,82 \\
\hline $\mathrm{abr} / 12$ & 355067,20 & 35,73 & 2,15 & 1,85 & 7,96 & 5,57 & 6,12 & 0,71 \\
\hline $\mathrm{mai} / 12$ & 372994,20 & 35,03 & 2,06 & 1,99 & 4,41 & 5,52 & 6,11 & 0,74 \\
\hline jun/12 & 374861,10 & 35,13 & 2,07 & 2,05 & 0,96 & 5,38 & 6,22 & 0,64 \\
\hline $\mathrm{jul} / 12$ & 366886,60 & 34,92 & 2,10 & 2,03 & 5,28 & 5,58 & 6,20 & 0,68 \\
\hline ago/12 & 374748,70 & 35,23 & 1,87 & 2,03 & 5,03 & 5,68 & 6,21 & 0,69 \\
\hline set/12 & 355521,00 & 35,49 & 1,83 & 2,03 & 7,06 & 5,64 & 6,37 & 0,54 \\
\hline out/12 & 382500,10 & 35,39 & 1,75 & 2,03 & 7,31 & 5,47 & 6,30 & 0,61 \\
\hline nov/12 & 394760,10 & 35,09 & 2,00 & 2,07 & 7,44 & 5,36 & 6,60 & 0,55 \\
\hline $\mathrm{dez} / 12$ & 390416,30 & 35,21 & 1,39 & 2,08 & 9,90 & 5,63 & 7,40 & 0,55 \\
\hline
\end{tabular}


Tabela A-1 - Variáveis utilizadas no modelo (continuação)

\begin{tabular}{lcccccccc}
\hline \multicolumn{1}{c}{ PERÍODO } & PIB & DIVIDAPIB & \multicolumn{1}{c}{ SP } & CAMBIO & IPCA & EXPIPCA & M1 & SELIC \\
\hline jan/13 & 382294,80 & 35,17 & 1,22 & 2,03 & 10,82 & 5,70 & 6,48 & 0,60 \\
fev/13 & 361731,60 & 35,61 & 1,52 & 1,97 & 7,44 & 5,50 & 6,43 & 0,49 \\
mar/13 & 372090,00 & 35,63 & 1,60 & 1,98 & 5,79 & 5,53 & 6,54 & 0,55 \\
abr/13 & 394148,30 & 35,48 & 1,73 & 2,00 & 6,80 & 5,56 & 6,36 & 0,61 \\
mai/13 & 408991,00 & 34,81 & 1,77 & 2,03 & 4,53 & 5,62 & 6,44 & 0,60 \\
jun/13 & 414747,00 & 34,50 & 1,85 & 2,17 & 3,17 & 5,74 & 6,53 & 0,61 \\
jul/13 & 406821,40 & 34,08 & 1,94 & 2,25 & 0,36 & 5,66 & 6,35 & 0,72 \\
ago/13 & 409429,60 & 33,85 & 2,14 & 2,34 & 2,92 & 6,06 & 6,38 & 0,71 \\
set/13 & 397189,20 & 34,69 & 2,46 & 2,27 & 4,28 & 6,16 & 6,39 & 0,71 \\
out/13 & 404233,00 & 34,94 & 2,51 & 2,19 & 7,06 & 6,25 & 6,28 & 0,81 \\
nov/13 & 417285,10 & 33,91 & 1,95 & 2,30 & 6,68 & 6,14 & 6,61 & 0,72 \\
\hline
\end{tabular}

Fonte: Elaboração própria. 